\title{
GT2019-90450
}

\section{HEAT TRANSFER PREDICTION FROM LARGE EDDY SIMULATION OF A ROTATING CAVITY WITH RADIAL INFLOW}

\author{
Michel Onori, Dario Amirante, Nicholas J. Hills and John W. Chew \\ Thermo-Fluid System UTC \\ University of Surrey \\ Guildford, Surrey, GU2 7XH, UK \\ Email:m.onori@surrey.ac.uk
}

\section{ABSTRACT}

The paper describes a Large Eddy Simulation (LES) conducted for a non adiabatic rotating cavity with a radial inflow introduced from the shroud. The dimensionless mass flow rate of the radial inflow is $C_{w}=3500$ and the rotational Reynolds number, based on the cavity outer radius, is equal to $\operatorname{Re}_{\theta}=$ $1.2 \times 10^{6}$. The time averaged local Nusselt number on the heated wall is compared with the experimental data available from the literature, and with those derived from the solution of two Unsteady Reynolds Averaged Navier-Stokes (URANS) eddy viscosity models, namely the Spalart-Allmaras and the $k-\omega S S T$ model. It is shown that the Nusselt number is underpredicted in the lower part of the disc and over-predicted in the outer region by both URANS models, whereas the LES provides a much better agreement with the measurements. The behaviour results primarily from a different flow structure in the source region, which, in the LES, is found to be considerably more extended and show localized buoyancy phenomena that the URANS models investigated do not capture.

\section{NOMENCLATURE}

$$
\begin{array}{ll}
a & \text { inner cavity radius } \\
b & \text { outer cavity radius } \\
C_{w}=\dot{m}_{r} / \mu b & \text { mass flow rate coefficient } \\
k^{*}=\frac{1}{2} \overline{u_{i}^{\prime} u_{i}^{\prime}} /(\Omega b)^{2} & \text { nondimensional turbulent } \\
& \text { kinetic energy }
\end{array}
$$

$$
\begin{array}{ll}
M_{\theta}=\Omega b / \sqrt{\gamma R T_{0}} & \text { rotational Mach number } \\
\dot{m}_{r} & \text { mass flow rate } \\
N u=\frac{\dot{q} r}{k\left(T_{w}-T_{0}\right)} & \text { Nusselt number } \\
q & \text { computed heat flux } \\
R e_{\theta}=\Omega b^{2} / v & \text { rotational Reynolds number } \\
R o=\bar{v}_{r} / \Omega b & \text { Rossby number } \\
r^{*}=r / b & \text { nondimensional radius } \\
S=V_{\theta} / \Omega r & \text { swirl ration in the absolute } \\
& \text { frame of reference } \\
s & \text { axial spacing between the discs } \\
T_{0} & \text { inlet total temperature } \\
T^{*}=T / T_{0} & \text { nondimensional fluid } \\
& \text { temperature } \\
u_{i}^{\prime} & \text { fluctuating flow component of the } \\
& \text { resolved scales in the Cartesian } \\
& \text { frame of reference } \\
V_{r}, V_{\theta}, V_{z} & \text { velocity components } \\
V_{r}^{*}=V_{r} / \Omega b, V_{\theta}^{*}=V_{\theta} / \Omega b & \text { nondimensional velocity } \\
& \text { components relative to } \\
& \text { the absolute frame of reference } \\
v_{r}, v_{\theta}, v_{z} & \text { velocity component relative to } \\
& \text { the frame of reference } \\
\bar{v}_{r} & \text { co-rotating with the cavity } \\
z^{*}=(z-\bar{z}) / s & \text { mean radial velocity } \\
& \text { at the inflow } \\
& \text { nondimensional axial coordinate }
\end{array}
$$




\author{
GREEK \\ $\varepsilon \quad$ turbulent dissipation rate \\ $\varepsilon_{w} \quad$ weighting coefficient in the Roe flux \\ $\mu \quad$ fluid viscosity \\ $v=\mu / \rho \quad$ fluid kinematic viscosity \\ $\rho \quad$ fluid density \\ $\Omega \quad$ angular speed
}

\section{INTRODUCTION}

The internal geometry of an high pressure compressor consists of a series of rotating disc cavities confined at the lower radius by the drive shaft. Cooling air is generally extracted from the intermediate stages of the compressor, and routed into the annular passage between the shaft and the discs (Fig. 1). This air is used for rim sealing and cooling in the later stages of the turbine. The axial throughflow of cooling air may interact with the flow inside the cavities and affect the temperature of the compressor discs. An accurate prediction of the disc temperature is important to determine the thermal stresses and the thermal distorsion of the component. Thermal stresses influence the service life, while the radial growth of the disc is important for the design of the blade tip clearances [1].

The inter-disc cavities may be sealed, or, in some configurations, the shroud is equipped with holes supplying additional flow to the system. In the absence of radial inflow, the flow within the cavity is approximately in solid body rotation, i.e. it rotates at the disc angular speed. In this case, the heat transfer on the internal surfaces is weak and dominated by buoyancy effects resulting from thermal stratification. In an engine, during the cruise or deceleration phase of the flight cycle, the shroud and the discs are typically hotter than the axial throughflow, and the centrifugal force drives colder fluid into the cavity through elongated radial arms. The flow field may exhibit large counter-rotating vortices (cyclonic and anti-cyclonic regions) with their axis parallel to the axis of rotation. Evidence of these macro-structures can be found in the experiments of Farthing et al. [2], Bohn et al. [3], Günther et al. [4] or in the numerical simulations of Tian et al. [5], Sun et al. [6], PuttockBrown et al. [7] and Owen et al. [8].

When a small amount of radial inflow is introduced in the cavity from the shroud, the heat transfer mechanism is drastically changed. If the velocity of the injected fluid is small compared to the cavity rotation rate, the Coriolis force inhibits the radial penetration through the cavity centre [9], and the flow is diverted towards the solid walls, feeding two Ekmantype boundary layers that transport fluid to the inner region, as schemetically depicted in Fig. 2. This has been shown by smoke visualization in the experiments of Firouzian et al. [10], those of Farthing et al. [11] for the qualitatively similar case of radial outflow, and confirmed by a (surprisingly limited) num-

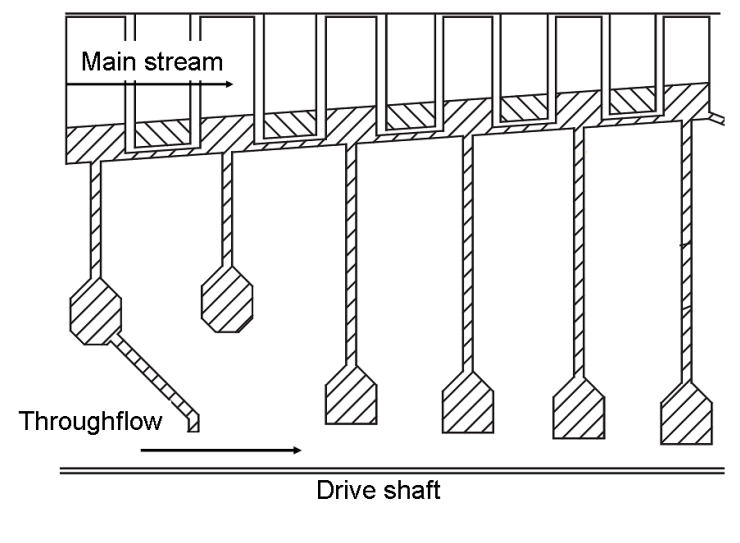

Figure 1. Schematic of a secondary air system in a compressor. From Childs [21].

ber of CFD studies [12-16].

Away from the walls, outside the Ekman layers, the flow is in a geostrophic condition, with the Coriolis force in equilibrium with the reduced pressure gradient. The flow develops along concentric streamlines in the cross-sectional $(r, \theta)$ plane, with the pressure gradient perpendicular to the flow direction. The tangential velocity grows at inner radii, and the reduced pressure gradient increases accordingly to balance the Coriolis force. In this area, buoyancy effects deriving from unstable thermal stratification are weak. In fact, the motion of the heavier fluid, driven into the cavity from the colder throughflow, is restrained by the augmented radial pressure gradient encountered within the cavity. For a sufficient amount of radial inflow, buoyancy effects are suppressed, or limited to the so-called "cob" region, i.e. the cavity restriction located just above the bore filled with the axial throughflow (Fig. 1). Inside the cavity, natural convection is replaced by forced convection, and the heat transfer on the surfaces is enhanced by the development of the Ekman layers. This has been shown in the parametric CFD study of Amirante et al. [15], in the conjugate analysis of Sun et al. [14], and in the experimental temperature measurements of Atkins [13] and Günther et al. [4].

The use of the radial inflow has been recently investigated as a possibility for compressor clearance control [13]. In a compressor, the design of the clearances between the blades and the main annulus casing has to take into account the centrifugal growth of the two components. Since the thermal response of the compressor drum is slower than that of the casing, the maximum blade tip clearance occurs immediately after acceleration, whereas the minimum clearance occurs immediately after deceleration. Clearly, the cold build clearances must be designed for the tightest clearance; this means that during 


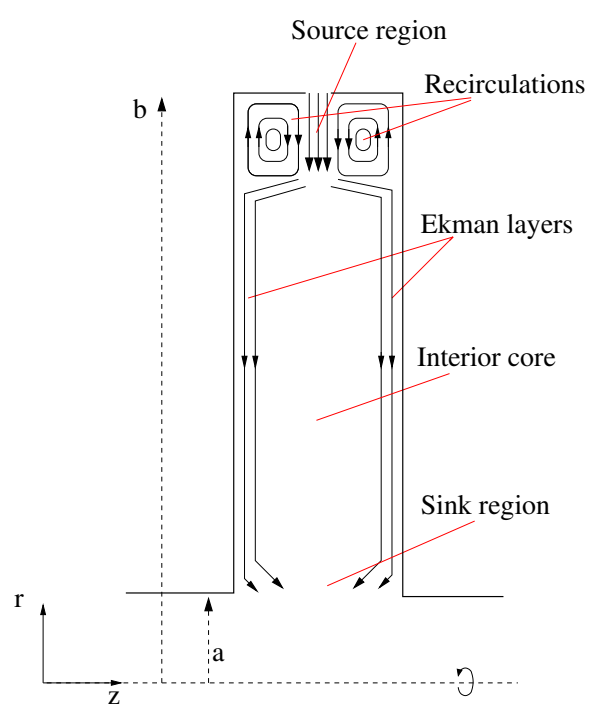

Figure 2. Schematic representation of the flow in a rotating cavity with radial inflow.

cruise the engine operates with larger clearances and has significant leakage around the blade tip. The radial inflow can be used to enhance the heat transfer on the discs, thereby reducing the mismatch in the thermal response between the compressor drum and the external casing and so leading to greater efficiency [13].

The aim of this study is to investigate the heat transfer prediction of both URANS and LES modeling for a compressor cavity with a radial bleed of air. The test case considered reproduces the experiment of Farthing et al. [17], in which a temperature profile is prescribed along one of the two discs, with the outer region hotter than the inner region. The radial inflow is colder than the wall, and the temperature in the Ekman layer grows rapidly while heat is released into the interior core. The process continues until the heat transfer on the surface is reversed.

The numerical simulations presented are validated comparing the computed Nusselt numbers with the experimental measurements. It will be shown that the URANS models employed, namely the $k-\omega S S T$ [18] and the Spalart-Allmaras [19] model, fail to capture the correct dynamics of the source region, and this leads to a misprediction of the Nusselt number. This behaviour, which is consistent with the numerical results of Kumar et al. [16], is substantially improved in the LES, and explained through a comparative analysis of the flow structure computed by the different models.

The layout of the paper is the following: in Section 2 we recall some theoretical considerations on the Ekman boundary layer, while a quick description of the CFD code is given in in Section 3. The CFD model set-up is given in Section 4 and

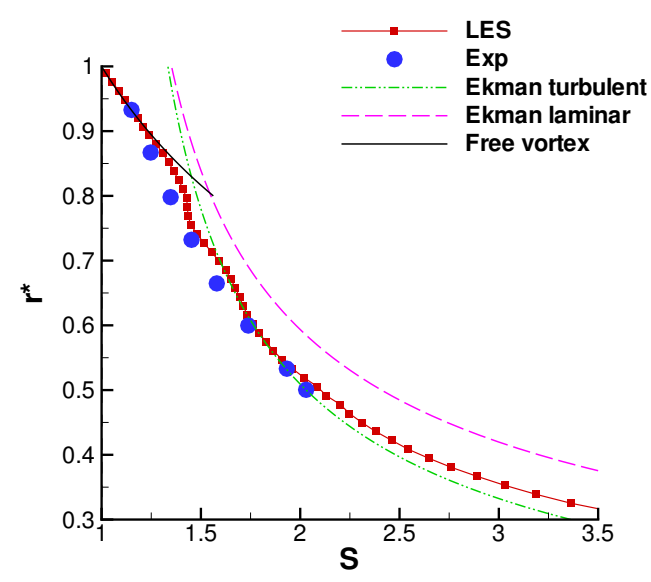

Figure 3. Radial profiles of swirl ratio $S=V_{\theta} / \Omega r$ for the isothermal test-case of Firouzian et al. [10].

the analysis of the results in Section 5. The main points of the study are finally summarized in the conclusions.

\section{ROTATING CAVITY WITH RADIAL INFLOW}

With reference to Fig. 2, we consider a cavity of radial extension $b-a$, rotating with angular speed $\Omega$ around the $z$ axis of a cylindrical frame of reference $(r, \theta, z)$. The velocity components relative to the frame of reference co-rotating with the cavity are denoted by $v_{r}, v_{\theta}, v_{z}$. The corresponding velocity components in the absolute frame of reference are $V_{r}, V_{\theta}, V_{z}$. The flow enters from the centre of the shroud with the same angular speed as the cavity, i.e. at $r=b, v_{\theta}=0$. The flow physics can be schematized diving the flow in three different regions: an inviscid core, Ekman boundary layers and a source region near the shroud, see Fig. 2. In the interior core, inertial and viscous terms are small compared to the Coriolis force, and the flow is said to be geostrophic. No penetration occurs $\left(v_{r}=0\right)$ and $v_{\theta}$ is the only non-zero velocity component [9].

Near the wall, the balance of forces must also involve the viscous effects, causing a departure from geostrophic conditions. Following the usual boundary layer approximations and linearizing with the assumption that $v_{r}, v_{\theta}, v_{z}<<\Omega r$, with a few manipulations one obtains the Ekman equations

$$
\begin{gathered}
-2 \rho \Omega\left(v_{\theta}-v_{\theta}^{\infty}\right)=\frac{\partial \tau_{r}}{\partial z} \\
2 \rho \Omega v_{r}=\frac{\partial \tau_{\theta}}{\partial z}
\end{gathered}
$$

where $v_{\theta}^{\infty}$ is the relative tangential velocity in the core and the 


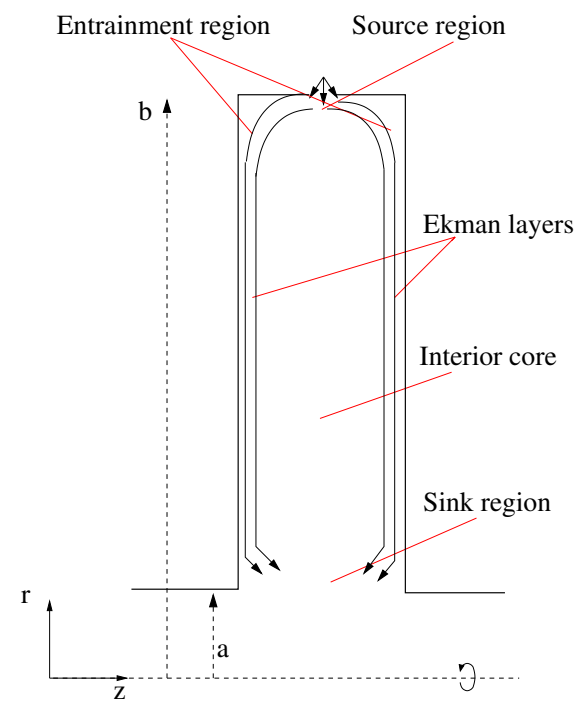

Figure 4. Schematic representation of the flow in a rotating cavity with radial infow, with inlet swirl ration $S(b)=1$.

two stress components are given by:

$$
\begin{aligned}
\tau_{r} & =\left(\mu+\mu_{t}\right) \frac{\partial}{\partial z} v_{r} \\
\tau_{\theta} & =\left(\mu+\mu_{t}\right) \frac{\partial}{\partial z} v_{\theta}
\end{aligned}
$$

If the flow is laminar, the Ekman equations can be integrated in closed form, and the resulting solution can be employed to obtain the core swirl ratio. Denoting by $S=V_{\theta} / \Omega r$ the swirl ratio in the absolute frame of reference, this gives $[20,21]$

$$
S=1+\frac{1}{2 \pi} \frac{C_{w}}{\operatorname{Re}_{\theta}^{1 / 2}}\left(\frac{r}{b}\right)^{-2}
$$

where $C_{w}=\dot{m}_{r} / \mu b$ is the nondimensional mass flow rate. For the turbulent case, it is possible to use an integral-momentum theory similar to that used by Von Kármán to solve the equations of the free disc. This gives [20]:

$$
S=1+2.22 \frac{C_{w}^{5 / 8}}{\operatorname{Re}_{\theta}^{1 / 2}}\left(\frac{r}{b}\right)^{-13 / 8}
$$

Figure 3 shows the swirl ratio obtained experimentally by Firouzian et al. [10] for an isothermal cavity at the conditions
$\operatorname{Re}_{\theta}=3.45 \times 10^{5}$ and $C_{w}=1300$. In the same figure, we report the solutions corresponding to Eq. 3 and Eq. 4, along with that from an LES conducted by the current authors in a previous study [22]. For nondimensional radii $r^{*}=r / b$ smaller than $r^{*} \simeq 0.9$, the experimental data are well matched by the turbulent Ekman layer solution, despite the fact that the relative tangential velocity $v_{\theta}$ is not small compared to the rotational speed of the disc. Owen et al. [23] obtained solutions of the nonlinear set of boundary layer equations, using an integral method for several cases in the laminar and turbulent regime. It was found that Eq. 3 and 4 provide reasonably accurate results even when the swirl ratio is significantly greater than one.

The Ekman layer solutions are valid away from the shroud; at outer radii, there has to be a region where the radial velocity in the core cannot be equal to zero, in order to account for the radial inflow penetration. This is generally referred to as the source region. Here, a possible is the free vortex $r V_{\theta}=$ const. In fact, any variation in the angular momentum of the fluid, $L=$ $r V_{\theta}$, corresponds to work done by the tangential component of the shear stress on the walls. If the flow enters in solid body rotation, the moment exerted by the shroud is zero, and the angular momentum can change only if differential rotation is established near the discs. According to the Ekman equations, this is possible only if there exist shear forces associated with flow in the radial direction (see the first of Eq. 1). In other words, as long as $v_{r}=0$ in the boundary layer, $\Delta L$ has to be equal to zero in the core, i.e. the free vortex solution.

The case where the radial inflow has a smaller rotational rate than the discs, is different. Eq. 1, along with Eq. 2, implies that when $v_{\theta}>v_{\theta}^{\infty}$ the curvature of the radial velocity profile $v_{r}$ is negative, i.e. $\frac{\partial^{2} v_{r}}{\partial z^{2}}<0$, and corresponds to outflow in the boundary layer. The schematic of Fig. 2 refers to this case: the Coriolis force near the wall drives the flow radially outwards against the pressure gradient. Mechanical work is done by the disc to increase the angular momentum of the fluid, and the two regions with recirculating flow extend until the core tangential velocity exceeds the speed of the disc. If the inlet swirl ratio is equal to 1 , the recirculating regions disappear, and the jet starts being gradually entrained into the boundary layer as soon as it enters the cavity, as depicted in Fig. 4. The swirl ratio departs from the free vortex, and becomes closer to the Ekman layer solution, as confirmed in Fig. 3 for the test-case of Firouzian.

In the experiments of Farthing et al. [11, 17], tests were conducted for $900 \leq C_{w} \leq 14000$ and $0.16 \times 10^{6} \leq \operatorname{Re}_{\theta} \leq$ $2 \times 10^{6}$ and the measured distribution of the Nusselt number was compared with the predictions of an integral method. They found that the Nusselt number reaches a maximum value where the integral method predicts the edge of the source region, i.e. where the entrainment is completed. However, the agreement between measurements and predictions was satisfactory only outside the source region, to the point that the authors ex- 
pressed doubts on the validity of the measurements near the shroud. It is anticipated that the computations presented in this paper confirm the experimental data of Farthing et al., at least for the specific case considered here.

\section{NUMERICAL METHOD}

The simulations presented in this paper have been conducted using the Rolls-Royce proprietary code Hydra. Hydra is a vertex-based, unstructured finite-volume solver of the compressible Navier-Stokes equations. Details of the numerical method can be found in references $[24,25]$. Here we limit to say that the spatial discretization is based on a second-order MUSCL scheme [26]; the convective flux on the surface of the control volume is approximated using the Riemann solver of Roe and consists of an inviscid and dissipative contribution:

$$
\mathbf{F}^{C} \cdot \mathbf{n} \simeq \mathbf{H}^{I} \cdot \mathbf{n}+\mathbf{H}_{\mathbf{n}}^{D}
$$

where $\mathbf{n}$ is the normal to the surface. Denoting by $L$ and $R$ the left and right states at any integration point, the numerical fluxes are computed as

$$
\begin{aligned}
& \mathbf{H}^{I} \cdot \mathbf{n}=+\frac{1}{2}\left(\mathbf{F}_{L}^{C}+\mathbf{F}_{R}^{C}\right) \cdot \mathbf{n} \\
& \mathbf{H}_{\mathbf{n}}^{D}=-\varepsilon_{w} \frac{1}{2}\left|\mathbf{A}_{\mathbf{n}}\right|\left(\mathbf{W}_{R}-\mathbf{W}_{L}\right)
\end{aligned}
$$

in which $\mathbf{A}_{\mathbf{n}}$ is the Roe matrix associated with $\mathbf{n}$.

The resulting scheme has a leading term of the truncation error proportional to the third spatial derivative, rendering the scheme predominantly dispersive. The numerical dissipation is third-order and proportional to the fourth spatial derivative. The weighting coefficient $\varepsilon_{w}$ appearing in the second of Eq. 6 can be used to calibrate the dissipative flux. Following common practice, the coefficient $\varepsilon_{w}$ is reduced to the minimum value necessary to preserve the stability of the computation.

In the LES, the WALE (Wall Adapting Local Eddy) model of Nicoud and Ducros [27] is adopted as subgrid scale (SGS) term, and the time integration is carried out using an explicit Runge-Kutta three-stage method [24]. The WALE model is preferred to the classical Smagorinsky closure as it provides the correct scaling of the subgrid eddy viscosity near the wall. In addition, the WALE model is designed for flows with both large strain rates and large rotational rates [27]. In the URANS, a Dual Time Stepping approach is used for time integration, and the additional transport equations employed for the Spalart-Allmaras and the $k-\omega S S T$ model follow the formulation given by Mariani et al. [28] and Menter [18], respectively. Previous work from Kumar et al. [16], carried out using

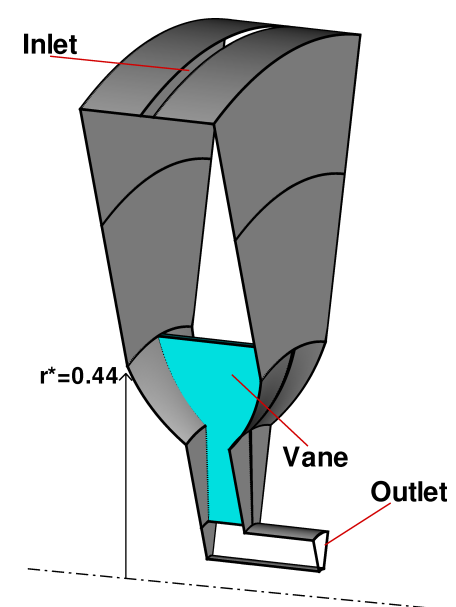

Figure 5. Computational domain employed in the study. The wall on the left is heated for $r^{*}>0.44$.

the same code Hydra, has shown that the $k-\varepsilon$ model is not adequate for this class of flows, and therefore it has not been considered in this study.

Particular care has to be taken for the treatment of the outlet boundary conditions. While in the URANS a pressure outlet can be safely adopted, in the LES it is necessary to use non-reflective boundary conditions to ensure that the turbulent structures can leave the domain without spurious oscillations. In this work we have used the local one-dimensional inviscid (LODI) approach of Poinsot and Lele [29].

\section{LES AND URANS MODEL SET-UP}

The computational domain is shown in Fig. 5. The cavity is confined between two lateral discs and a peripheral shroud. The inner and outer radius are $a=12 \mathrm{~mm}$ and $b=381 \mathrm{~mm}$, respectively. The distance between the discs is $s=102 \mathrm{~mm}$. A central vane is positioned in the cob region and extending up to $r^{*}=0.44$, where $r^{*}$ denotes the nondimensional radius $r^{*}=r / b$. The model has a sector size of 36 degrees which corresponds, in the experimental facility, to the minimal periodic portion that includes a central vane.

The inlet boundary conditions employ a specified mass flow rate $\dot{m}_{r}=0.024 \mathrm{~kg} / \mathrm{s}$, a specified total temperature $T_{0}=$ $298 K$ (equal to the ambient temperature in the experiment) and a swirl ratio equal to $S=1$. Since the computation is performed in the rotating frame of reference, the latter condition is achieved imposing that the direction of the incoming flow is normal to the inlet surface. 
The rotational Reynolds and Mach numbers are:

$$
\begin{aligned}
& R e_{\theta}=\frac{\Omega b^{2}}{\nu}=1.3 \times 10^{6} \\
& M_{\theta}=\frac{\Omega b}{\sqrt{\gamma R T_{0}}}=0.15
\end{aligned}
$$

The Rossby number, which measures the relative importance of the radial inflow over the Coriolis force, is

$$
R o=\frac{\bar{v}_{r}}{\Omega b}=7.6 \times 10^{-3}
$$

where $\bar{v}_{r}$ is the mean velocity at the inflow. The nondimensional mass flow rate is:

$$
C_{w}=\frac{\dot{m}_{r}}{\mu b}=3500
$$

There is experimental evidence $[10,23]$ that transition in the boundary layer occurs when $C_{w} \simeq 1200$. Hence, in the current problem the Ekman layers are expected to be fully turbulent. A temperature profile is prescribed on the heated disc (the one on the left in Fig. 5), between the upper end of the cob region $\left(r^{*}=0.44\right)$ and the periphery $\left(r^{*}=1\right)$. The ratio between the maximum temperature assigned on the wall $T_{w}^{M}$, and the inlet total temperature $T_{0}$, is equal to 1.15 ; the buoyancy parameter is $\alpha\left(T_{w}^{M}-T_{0}\right)=0.16$, where $\alpha$ is the volumetric expansion coefficient of the inlet fluid. The precise distribution of the disc temperature is shown in Fig. 14 and will be discussed in the results section. All the other walls, including the shroud, the vane and the disc on the right, are adiabatic.

An averaged URANS solution has been used to inizialize the LES calculation. Therefore both the LES and URANS simulations presented have been conducted on a mesh with 15 million hexahedral cells with the near wall grid spacing $y^{+} \simeq 1$ throughout the cavity. URANS on a coarser mesh $(\sim 1.5$ million cells) have given identical results. Following Davidson [30], an adequate mesh resolution in wall resolved LES requires approximately 100 and 30 wall units in the streamwise and spanwise direction, respectively. In our case it was impossible to fulfill the above requirements everywhere, as we had to comply with the available computational resources. The meshing strategy was aimed at resolving very well the upper part of the cavity, where the Nusselt number prediction might be critical for the development of the source region. Figure 6 shows the variation along the radius of $\Delta r^{+}, \Delta z^{+}$and $\Delta(r \theta)^{+}$at the cavity mid-axial position. The curves have been obtained using the wall units calculated at each radius from $\theta$-averaged values of the shear stress on the wall. In the Ekman layer the

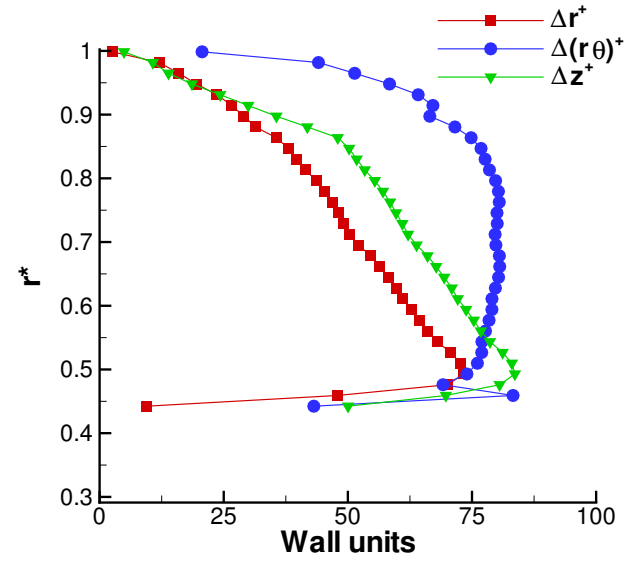

Figure 6. Radial profiles of nondimensional grid spacing at the cavity centre, resulting from the LES computation.

flow is predominantly oriented in the tangential direction; according to Fig. 6, while the overall streamwise resolution falls in the range of recommended values, the spanwise resolution is less satisfactory and degrades at lower radii. Computations on finer meshes have not been considered, because the physical time scale of the problem is too long. Starting from a URANS solution, statistical convergence in the LES presented has been achieved after approximately 16 disc revolutions. Each of them has taken almost 6 days running on 20 nodes of a Linux Cluster, each node consisting of 16 Intel Xeon processors sharing 32 GB of memory.

\section{LES AND URANS RESULTS}

The validation of the results against the experimental distribution of the Nusselt number is presented after an initial general description of the flow structure. For both LES and URANS the profiles presented have been extracted from averages of the mean flows in the circumferential direction. A single mean flow results from the time average of the instantaneous flow field over one disc revolution. The nondimensional radius is $r^{*}=r / b$, while the axial profiles are plotted against $z^{*}=(z-\bar{z}) / s$, where $\bar{z}$ is the axial coordinate at the cavity centre, and $s$ the cavity width. The velocity components are divided by the velocity of the disc at the periphery $\Omega b$, and denoted by $V^{*}$. Similarly, the fluid temperature is nondimensionalized using the inflow total temperature and denoted by $T^{*}$. 


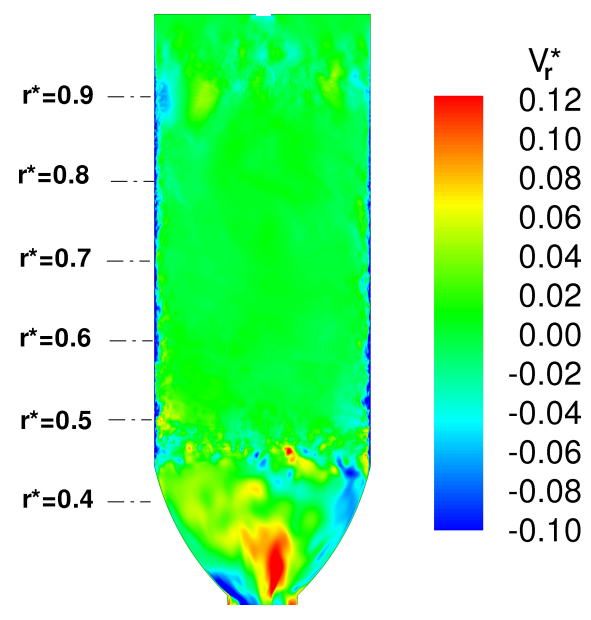

Figure 7. LES instantaneous flow field. Contour plot of radial velocity component $V_{r}^{*}=V_{r} / \Omega b$ on a meridional plane.

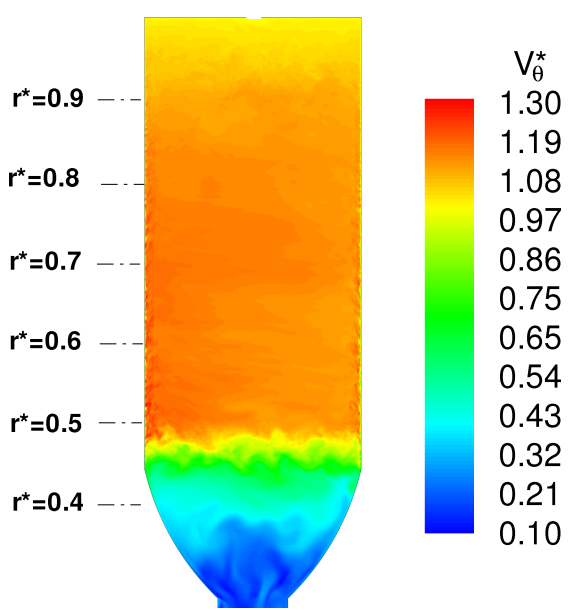

Figure 8. LES instantaneous flow field. Contour plot of tangential velocity component $V_{\theta}^{*}=V_{\theta} / \Omega b$ on a meridional plane.

\section{LES flow field}

LES instantaneous velocity contours in a meridional plane are shown in Fig. 7 and 8. There is a large core separating two turbulent boundary layers convecting the fluid inward. Little activity is present in the core, at least for $0.5<r^{*}<0.9$. The radial velocity remains very small while the tangential velocity varies predominantly along the radius, although careful inspection of Fig. 8 reveals slightly higher values near the heated wall. A more chaotic behaviour can be observed in the cavity restriction, with the appearance of smaller turbulent structures. Here the tangential velocity is drastically reduced due to the presence of the vane that induces solid body rotation to the fluid.

Fig. 9 shows the turbulent kinetic energy associated with

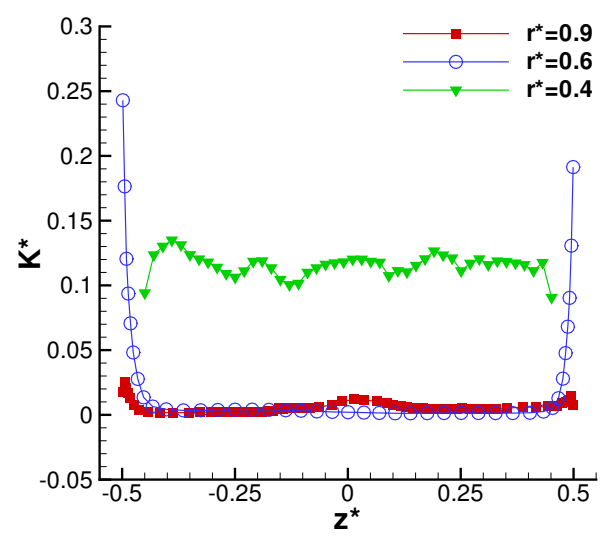

Figure 9. Axial profiles of turbulent kinetic energy $k^{*}=\frac{1}{2} \overline{u_{i}^{\prime} u_{i}^{\prime}} /(\Omega b)^{2}$ at different radial positions.

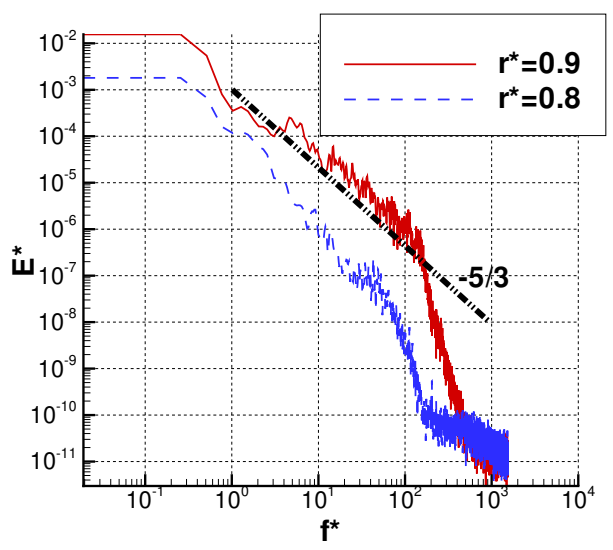

Figure 10. Energy spectra of the velocity fluctuations at the cavity centre. Frequencies are nondimensionalized by $2 \pi / \Omega$.

the resolved scales, $k^{*}=\frac{1}{2} \overline{u_{i}^{\prime} u_{i}^{\prime}} /(\Omega b)^{2}$, at different radii. To enable comparison with the velocity field it is possible to refer to Fig. 7 and 8. Away from the vanes, turbulence grows in the boundary layers while the core remains relatively quiescent. The turbulent kinetic energy $k^{*}$ is equal in the core to 0.012 at $r^{*}=0.9,0.002$ at $r^{*}=0.6$ and 0.12 at $r^{*}=0.4$. It is also interesting to note the asymmetric behaviour in the two boundary layers, with $k^{*}$ slightly higher near the hot wall. Fig. 10 shows the energy spectra of velocity fluctuations registered in the cavity centre at $r^{*}=0.9$ and $r^{*}=0.8$. The spectra at $r^{*}=0.9$ exhibits a $-5 / 3$ slope extending in the range $1<f^{*}<100$, and a clear cut-off is visible at $f^{*} \simeq 100$. This suggests that the resolution employed is sufficient. At $r^{*}=0.8$, the energy content is significantly smaller, as the effect of the jet is less important and the rotation tends to stabilize the flow in the core. 


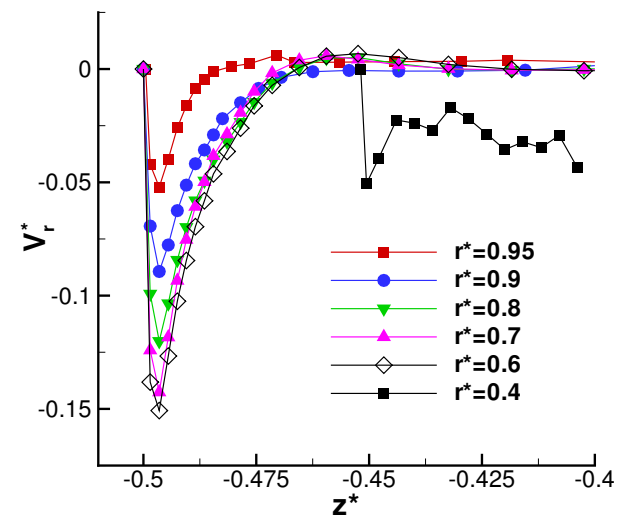

Figure 11. Axial profiles of radial velocity component $V_{r}^{*}=V_{r} / \Omega b$ at different radial positions.

LES axial profiles of radial velocity are shown in Fig. 11. The axial coordinate of the figure extends from the heated wall for $10 \%$ of the cavity width (from $z^{*}=-0.5$ to $z^{*}=-0.4$ ). The corresponding values of mass flow rate $\dot{m}^{*}=\dot{m} / \dot{m}_{r}$, obtained by integrating the boundary layer profiles, are shown in Fig. 12, It can be seen that in the LES solutions the entrainment is completed at $r^{*} \simeq 0.9\left(\dot{m}^{*} \simeq 0.5\right)$ and then $\dot{m}^{*}$ reaches a maximum at $r^{*} \simeq 0.85$. Here the mass flow rate in the boundary layer is larger than the nominal values $\dot{m}_{r} / 2$. For $r^{*}<0.85$, $\dot{m}^{*}$ reduces and becomes closer to $\dot{m}_{r} / 2$. According to Fig. 11, the mass flow rate reduction at inner radii results from the presence of small radial outflow located in the external part of the boundary layer.

At $r^{*}=0.4$, that is in the cob region, the radial penetration occurs through the core, and the Ekman layer disappears. The strong turbulence mixing and the acceleration induced by the cavity restriction, render the flow conditions no longer geostrophic. As we are mainly concerned with the heat transfer prediction in the cavity, no further analysis is reported for the cob region in the remainder of the paper.

The swirl ratio at the cavity mid-axial position is shown in Fig. 13 for the LES and URANS solutions. All the curves show a good agreement with the free vortex solution near the shroud, and exhibit a maximum located at the same position $r^{*}=0.5$. The predicted level of swirl, however, is significantly different. The $k-\omega S S T$ seems to be the least convincing solution. In fact, a lower level of swirl is indicative of a smaller amount of fluid pumped near the wall, with some of the radial inflow that moves through the core, cfr. Eq. 4.

As mentioned before, the LES is not fully resolved at lower radii. According to our previous study of an isothermal cavity [22], this may cause an overestimate of the swirl at inner

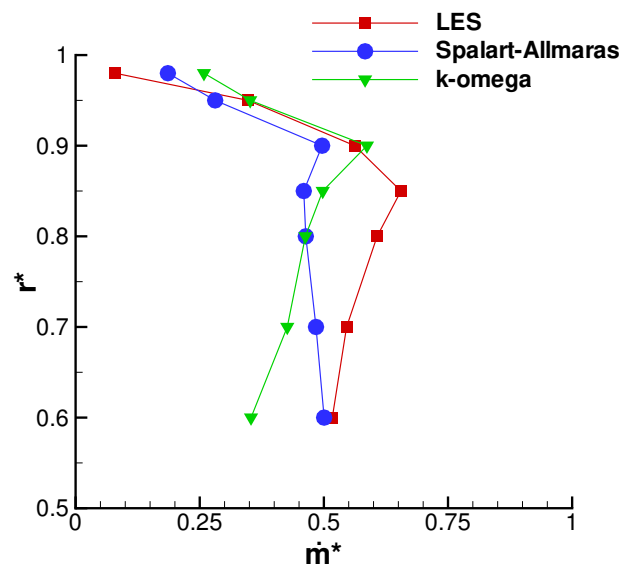

Figure 12. Mass flow rates computed across axial cuts adjacent to the heated wall, and extending for $10 \%$ of the cavity width. $\dot{m}^{*}=\dot{m} / \dot{m}_{r}$ where $\dot{m}_{r}$ is the radial inflow rate.

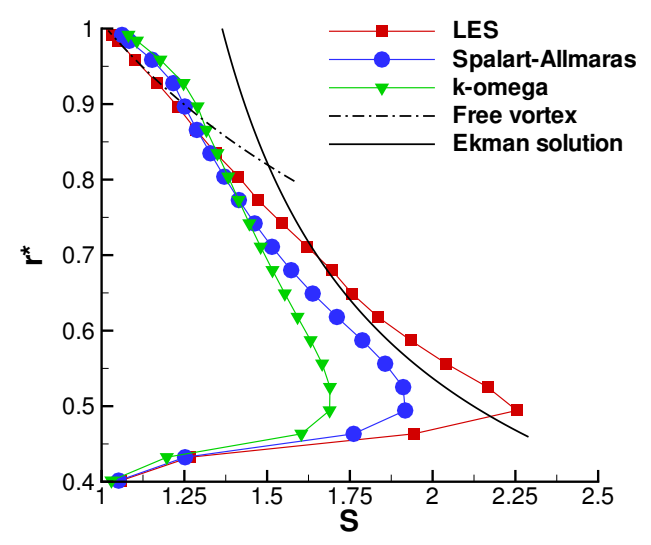

Figure 13. Radial profiles of swirl ratio $S=V_{\theta} / \Omega b$ at the cavity midaxial position.

radial positions, as the radial shear in the Ekman layers could be overpredicted. It may be instructive, in this context, to examine the difference between laminar and turbulent solution in Fig. 3. In our previous study [22], it has been shown that underresolved LES display a similar qualitative behaviour, with the swirl profile tending towards the laminar solution. However, Fig. 13 shows that the LES solution has a certain level of agreement with both the turbulent solution and the SpalartAllmaras model, indicating that a possible overestimation of the swirl in this case could be limited. This in turn suggests that the flow field could be reasonably accurate even at inner radii. 


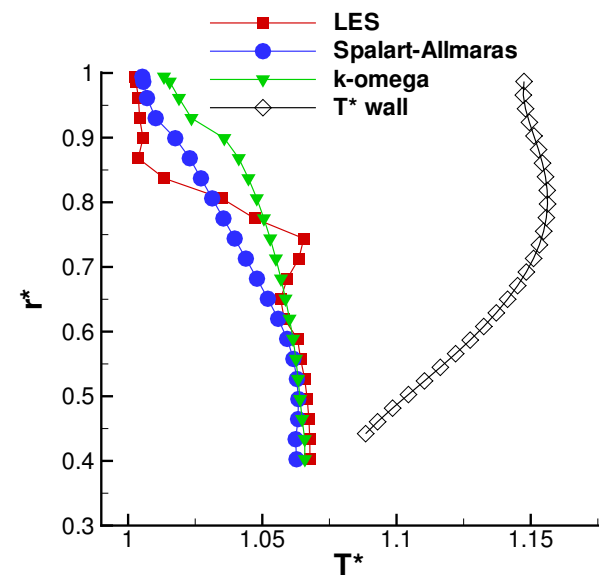

Figure 14. Wall temperature prescribed on the disc and radial profiles of temperature $T^{*}=T / T_{0}$ at the cavity mid-axial position.

\section{Heat transfer validation}

Radial temperature profiles at the cavity centre are given in Fig. 14, along with the prescribed wall temperature. The Nusselt number is evaluated as

$$
N u=\frac{\dot{q} r}{k\left(T_{w}-T_{0}\right)}
$$

where $\dot{q}$ is the computed heat flux, $k$ the conductivity of the air, and $T_{w}-T_{0}$ the difference between wall temperature and inlet total temperature. Figure 15 compares the time-averaged local Nusselt number with the experimental measurements of Farthing et al. [17], reported with error bars corresponding to 95\% confidence intervals.

The agreement with the LES solution is good. Immediately below an initial overshoot located close to the shroud, the Nusselt number increases radially inwards, reaching a maximum at $r^{*} \simeq 0.85$. With a further decrease of the radius, the Nusselt number decreases and becomes negative at $r^{*}=0.57$, against the value $r^{*}=0.6$ measured in the experiment. The fluid temperature in the core remains approximately constant for $r^{*}>0.85$, and then increases until reaching a maximum at $r^{*} \simeq 0.7$. The behaviour observed at $r^{*} \simeq 0.7$, where the core temperature stops increasing, and the predicted curve of Nusselt number exhibits an inflectional point, is consistent with the experimental data distribution. These results provide confidence in the quality of the LES solution. As previously stated, the simulation has been performed by gradually reducing the weighting coefficient $\varepsilon_{w}$ of the dissipative flux. Fig. 16 shows the Nusselt number obtained for three different values of $\varepsilon_{w}$. It can be seen that while the solutions at inner radii are similar, the solution in the source region is drastically degraded

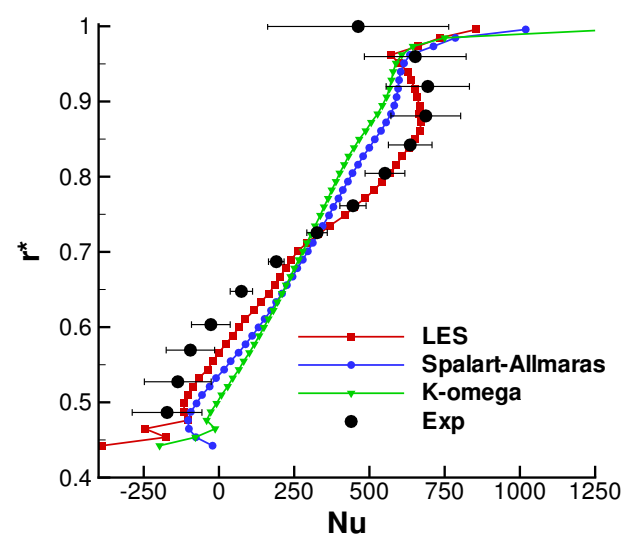

Figure 15. Variation of time-average local Nusselt numbers $N u$ on the heated wall with nondimensional radius $r^{*}=r / b$.

when using a relatively high value of $\varepsilon_{w}$. This result seems to confirm previously observations that LES solvers with secondorder schemes (in which the effect of the SGS model becomes important only within a few wall units from the wall [25]) may be too dissipative for the resolution generally employed in LES $[22,25]$. By reducing $\varepsilon_{w}$, the solution improves but becomes more prone to instability, because the dispersion error creates unphysical amplifications which are not sufficiently damped. The numerical dissipation of the current scheme (proportional to the $4^{\text {th }}$ spatial derivatives) does not incorporate the physics of an eddy viscosity model. Hence, it requires a resolution sufficiently high to achieve a clear separation of scales (as shown in the energy spectra of Fig. 10), so as to avoid that the energy is drained from the large structures. At inner radii, the effect of $\varepsilon_{w}$ appears less important. This is somehow expected, because the flow is fully turbulent and the heat flux depends primarily on the mass flow rate in the boundary layer, which is approximately the same once the entrainment is completed.

Both URANS models capture the general trend, but they overestimate the heat transfer in the inner region and underestimate it in the outer part. In particular, the peak observed in the data between $r^{*}=0.7$ and $r^{*}=0.9$ is completely missed. The Nusselt number distribution is essentially linear for $r^{*} \leq 0.95$, then exhibits a sudden increase, reaching a maximum at the periphery.

At a short distance from the inlet it is reasonable to assume that the effect of the heated wall on the jet is marginal. Hence, the rothalpy of the radial inflow, defined as $I=H-\Omega L$, where $H$ is the total enthalpy and $L=r V_{\theta}$, might expected to be initially constant. For $r^{*}>0.9$, in the source region, the flow behaves like a free vortex (Fig. 13). Thus, $I=$ const implies that $H=$ const. Since the Mach number is also small, compress- 


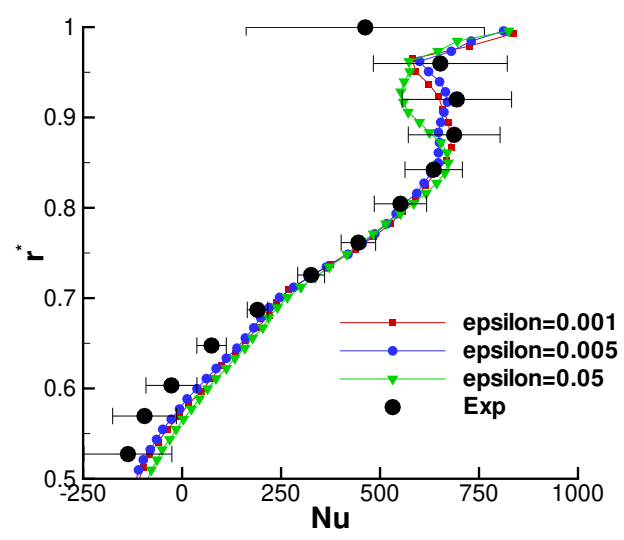

Figure 16. Effect of the numerical dissipation on the Nusselt number profiles along the heated wall.

ibility effects on a short radial extent cannot significantly alter the fluid temperature, which, therefore, has to remain approximately equal to the radial inflow temperature, as confirmed in Fig. 14. Outside the source region, for $r^{*} \leq 0.9$, the convection through the core is drastically reduced, and the temperature in the cavity centre begins to increase as the turbulent diffusion propagates heat from the wall.

Compared to the LES solution, the core temperature in the URANS remains constant for a shorter extent near the shroud, indicating a stronger effect of the turbulent diffusion over the radial convection. The next section will be devoted to analyzing closely the structure of the source region, which, based on the considerations developed, may be the main cause for the mis-prediction observed in the URANS models, as well as in the integral method solutions reported by Farthing et al. [17].

\section{Source region}

The flow structure in the source region is visualized in Fig. 17 and Fig. 18 for the Spalart-Allmaras and $k-\omega$ solution, respectively. Temperature contours in a meridional plane are superposed on the streamlines associated with the axial and radial velocity components. Both solutions show that the entering jet rapidly forms two small symmetric vortices adjacent to the jet. These structures are conceptually different from the regions of re-circulating fluid schematized in Fig. 2. Here they result from the shear forces acting between the entering jet and the fluid driven towards the shroud by the Coriolis force. The peak in the Nusselt number at $r^{*}=1$, observed in Fig. 15 reflects the presence of cold fluid that moves along the shroud before impinging on the hot disc at the periphery.

The streamlines develop symmetrically around the mid-

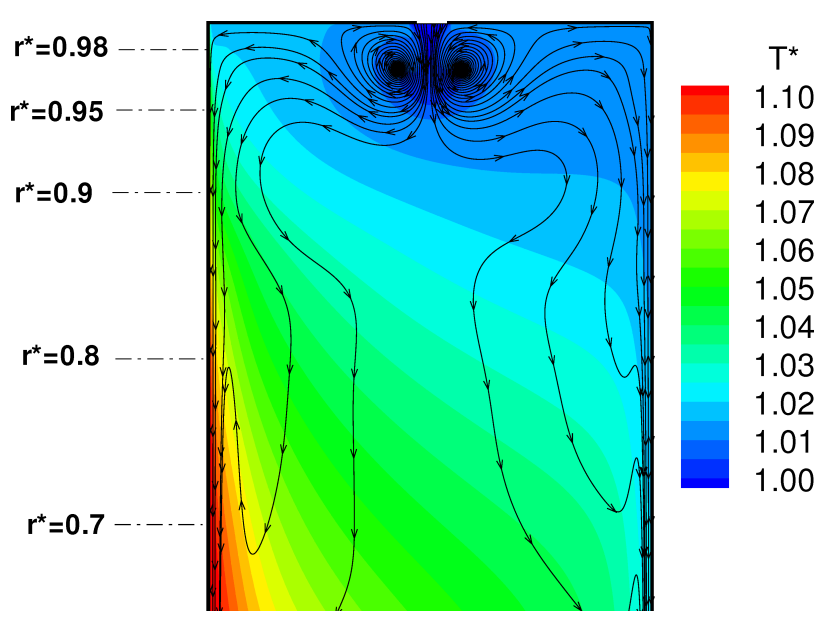

Figure 17. Spalart-Allmaras solution. Temperature contour plot and streamlines associated with the axial and radial velocity components.

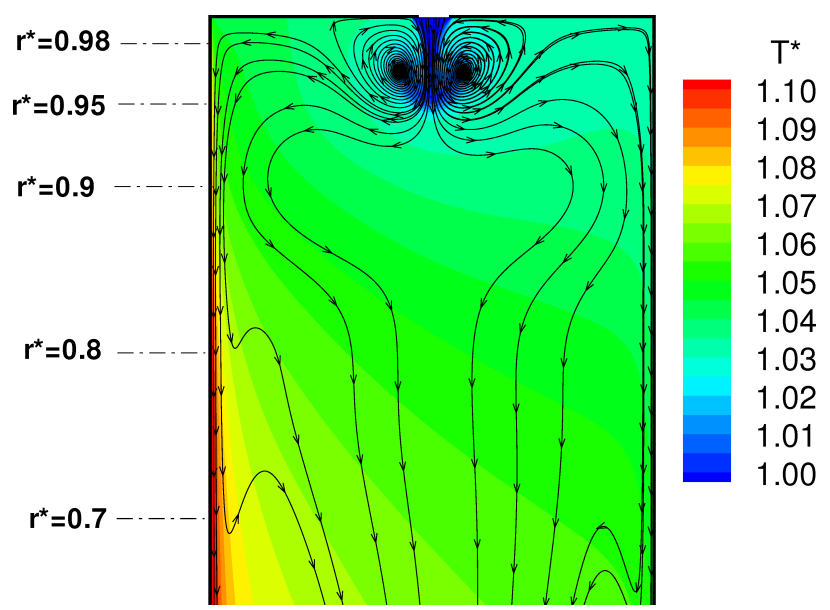

Figure 18. $k-\omega$ solution. Temperature contour plot and streamlines associated with the axial and radial velocity components.

axial position, and tend to cluster between $r^{*}=0.9$ and $r^{*}=$ 0.95 , suggesting that this is the region where the entrainment is completed. At about $r^{*} \simeq 0.9$, the streamlines rotate counterclockwise near the hot wall and clockwise near the cold wall, indicating the presence of fluid diverted from the two boundary layers towards the interior core. Consistent with this, the mass flow rate displayed in Fig. 12 shows a peak located exactly at $r^{*}=0.9$. This behaviour is predicted by both URANS models. However, in the $k-\omega$ solution some of the streamlines do not re-enter the boundary layers at inner radii, revealing that there is fluid moving through the core, in agreement with the indications given by the mass flow profiles of Fig. 12.

A snapshot of the LES thermal field is given in Fig. 19 showing contour plots on four $\theta$-planes. On each of them, an extended region can be seen where the fluid temperature 


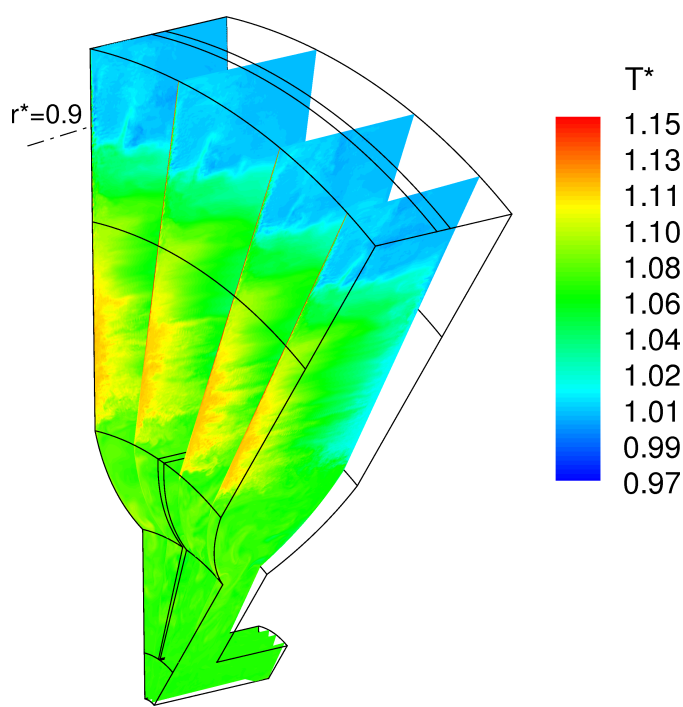

Figure 19. LES instantaneous solution. Temperature contours on different meridional planes.

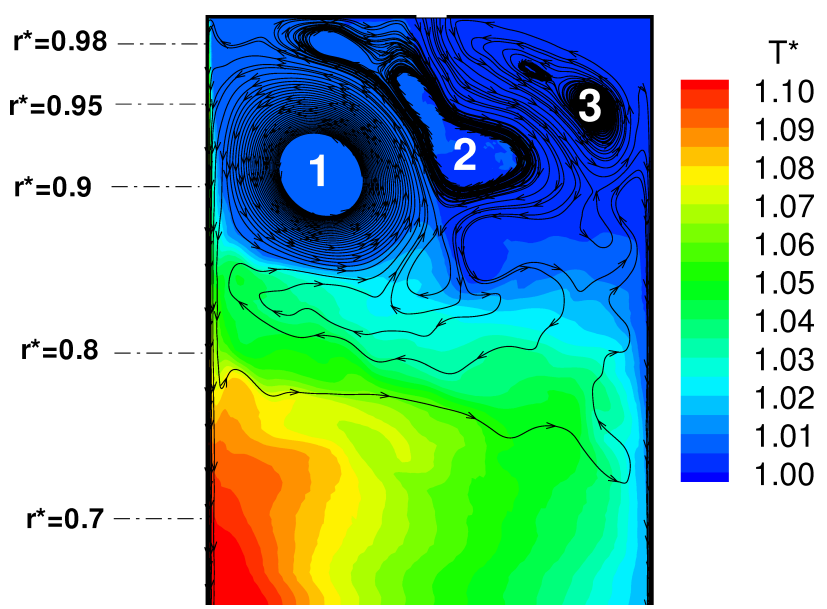

Figure 20. LES time averaged solution. Temperature contour plots and streamline associated with the axial and radial velocity component.

is fairly homogeneous and close to the radial inlet temperature. The selected contour levels are slightly different from Fig. 17 and 18, in order to show the small arms located near the hot wall at approximately $r^{*} \simeq 0.9$. The small arms depart from the edge of the source region and stretch outwards, as a result of buoyancy effects originating in the boundary layer.

As the flow is heated near the wall, a density gradient is formed in the axial direction, remaining disaligned from the pressure gradient. This induces baroclinic circulation in the meridional plane. In the vorticity equation, the baroclinic production term is given by $\frac{1}{\rho^{2}} \nabla \rho \times \nabla p$. Thus, the sign of the

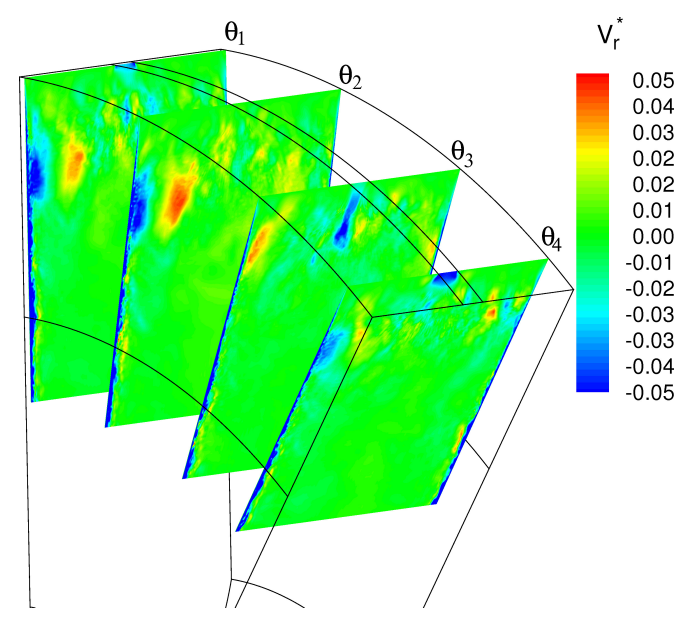

Figure 21. LES instantaneous solution. Contour plot of radial velocity component on different meridional planes.

azimuthal vorticity created near the hot wall induces counterclockwise circulation. On more physical grounds, buoyancy drives the flow heated near the wall below the heavier fluid of the stratified core. Figure 20 shows the streamlines corresponding to the time averaged flow on a specific $\theta$-plane. It can be noticed the radial inflow is deflected towards the cold disc. The vortices numbered by 2 and 3 should correspond to those also observed in the URANS solutions, adjacent to the jet. Vortex 1, on the other hand, is a buoyancy induced vortex. The angular momentum of the heated fluid is larger than that in the geostrophic core. Therefore, as the buoyant fluid moves away from the wall, it is also forced to move outwards, driven by the Coriolis force. In the cavity centre, just below Vortex 2 , it merges with an arm of the cold radial inflow, and then recirculates towards the wall, feeding the boundary layer. In this scenario, Vortex 1 represents the mechanism whereby the cold radial inflow is pumped near the hot wall.

It is useful to summarize, at this point of the discussion, the consistency among the various figures presented:

- The boundary layer mass flow rate increases drastically for $r^{*}<0.95$, reaching a maximum at $r^{*}=0.85$ (Fig. 12), corresponding to the upper and lower edge of Vortex 1 (Fig. 20).

- The Nussult number has a maximum value slightly above $r^{*}=0.85$, cfr. Fig. 15, corresponding to the lower edge of Vortex 1 in Fig. 20.

- Fluid temperature at the cavity centre is constant for $r^{*}>$ 0.85 (Fig. 14). For $r^{*}<0.85$ it increases faster than in the URANS solutions. Figure 20 shows that in this region the interior core is heated by natural convection.

Instantaneous radial velocity contours are shown in Figure 21 
for different $\theta$-planes. The flow field on the two planes $\theta_{1}$ and $\theta_{2}$ are consistent with the streamlines of Fig. 20, while plane $\theta_{3}$ shows fluid that moves in the opposite direction. A nonuniform distribution of $v_{r}$ at the inlet slot can also be observed. Animations of the phenomenon indicate that the flow behaves almost randomly near the cold wall, with the inlet conditions having to adjust locally to preserve the specified radial inflow rate. Vortex 1, on the other hand, appears to be remarkably robust, with small patches where it suddenly shrinks corresponding to localised radial outflow. The authors do not exclude that Vortex 1 could be the result of a combined effect. There might be, in fact, a tendency of the flow to move towards the interior core at the latest stages of the entrainment phase, as observed in both the URANS solutions. In the LES, buoyancy may reinforce this mechanism near the hot wall, eventually breaking the symmetry of the system.

\section{Eddy viscosity}

The last part of this paper is aimed at performing a comparative analysis between the eddy viscosity in the URANS models employed, and the turbulent viscosity predicted in the LES calculation.

In the Spalart-Allmaras model a transport equation is solved for the eddy viscosity $v_{t}$, whereas in the $k-\omega S S T$ model $\mathrm{v}_{t}$ is determined by the conventional KolmogorovPrandtl expression

$$
\mathrm{v}_{t}=\frac{k}{\omega}
$$

In the LES, one has available from the computation the turbulent kinetic energy $k$ and the turbulent dissipation rate $\varepsilon$. The latter is defined as

$$
\varepsilon=v \overline{\frac{\partial u_{i}^{\prime}}{\partial x_{k}} \frac{\partial u_{i}^{\prime}}{\partial x_{k}}}
$$

We point out that these quantities are associated with the resolved motion, and do not include the contribution of the subgrid scale model. A representative value of the corresponding eddy viscosity can then be given by the standard $k-\varepsilon$ relation [31]

$$
\mathrm{v}_{t}=C \frac{k^{2}}{\varepsilon}
$$

with $C=0.09$. In the Spalart-Allmaras computation, the eddy viscosity was specified at the inlet to be 10 times greater than the dynamic viscosity, i.e. at $r=b$ it is $v_{t} / v=10$ where $v=$

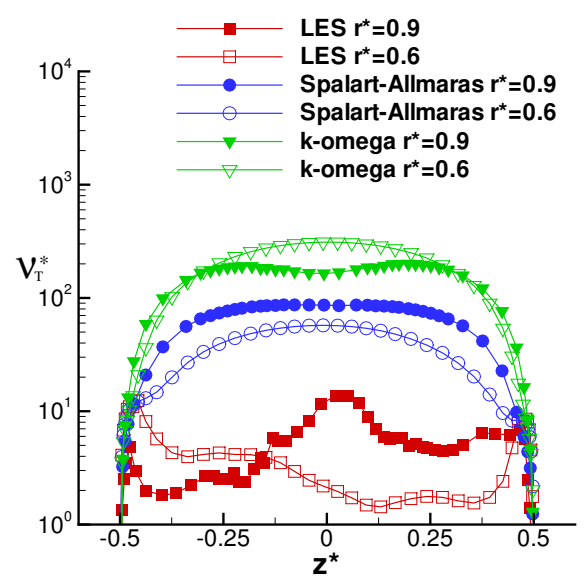

Figure 22. Axial profiles of turbulent viscosity $v_{t}^{*}=v_{t} / v$ at different radial positions.

$\mu / \rho$ is the kinematic viscosity of the radial inflow. In the $k-$ $\omega S S T$ computation, the kinetic energy $k$ was assigned through the specification of an inlet turbulence intensity equal to $10 \%$, and the value of $\omega$ was obtained enforcing the same viscosity ratio $v_{t} / v=10$. No perturbation was added at the inlet for the LES.

Axial profiles of turbulent viscosity are shown in Figure 22 for the two radial positions $r^{*}=0.6$ and $r^{*}=0.9$. In the cavity centre, at both radial locations, the Spalart-Allmaras solution exhibits a turbulent viscosity significantly greater than that calculated from the LES solution. In the $k-\omega S S T, v_{t}$ is between one and two orders of magnitude larger. The large disparity between the values computed at $r^{*}=0.9$ and those prescribed at the inlet, suggests very little sensitivity to the specified boundary conditions. Therefore, the turbulent kinetic energy in the source region has to be primarily associated with the presence of the jet. The behaviour at $r^{*}=0.6$ is less clear. Here, the URANS turbulent viscosity remains an order of magnitude larger than in the LES, while in the boundary layer the value of $\mathrm{v}_{t}$ becomes similar for the three models.

A sensitisation for rotation and curvature effects for the $k-\omega S S T$ model has been examined, following the $k-$ $\omega S S T R C$ model of Hellsten [32]. Compared to the standard $k-\omega S S T$ formulation [18], the destruction term in the $\omega$ equation is multiplied by a rotation and curvature correction factor $F_{4}$ introducing a modification of the turbulent length scale. The correction factor is

$$
F_{4}=\frac{1}{1+C_{r c} R_{i}}
$$

where $C_{r c}=3.6$ and the Richardson number $R_{i}$ is related to the 


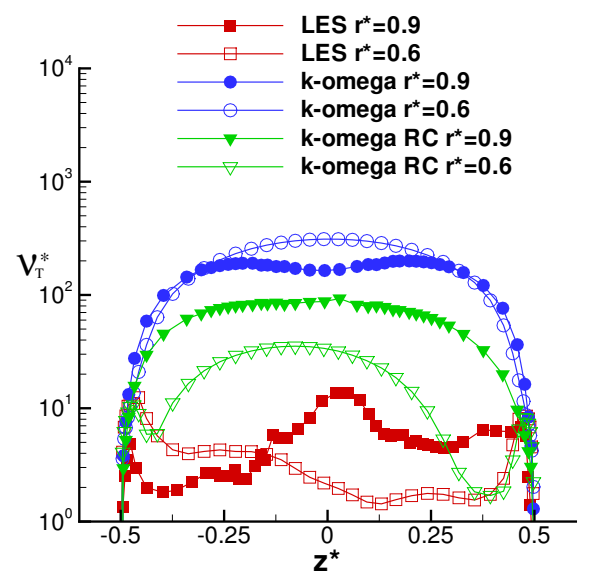

Figure 23. Sensitisation for rotation and curvature effects. Axial profiles of turbulent viscosity $v_{t}^{*}=v_{t} / v$ at different radial positions.

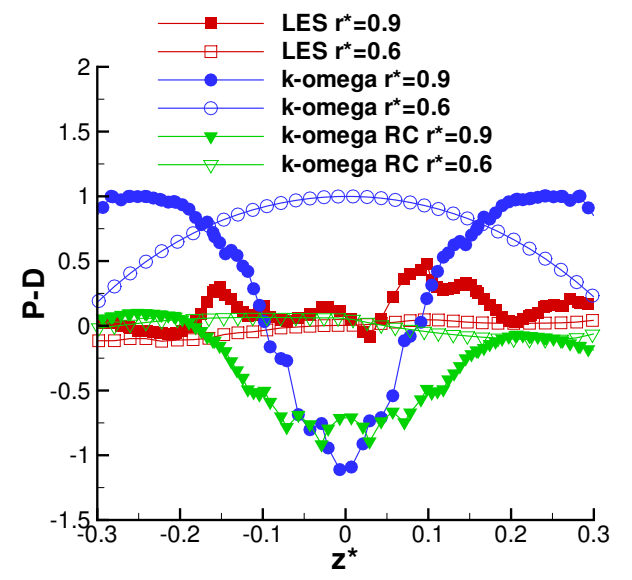

Figure 24. Sensitisation for rotation and curvature effects. Axial profiles of the normalized difference between the production and the destruction term of kinetic energy at different radial positions.

strain-rate tensor and the vorticity tensor [32].

The results obtained with the $k-\omega$ SSTRC model are shown in Fig. 23. The turbulent viscosity is compared with the standard $k-\omega S S T$ and LES solutions. The $R C$ correction reduces the turbulent viscosity which, at $r^{*}=0.6$, becomes one order of magnitude smaller than in the standard $k-\omega S S T$ model. Fig. 24 shows the axial profiles of the difference between the production $P$ and the destruction $D$ of turbulent kinetic energy. In the $k-\omega S S T$ and $k-\omega S S T R C$ models these quantities have been evaluated using the specific definitions of the terms, whereas in the LES the production term has been

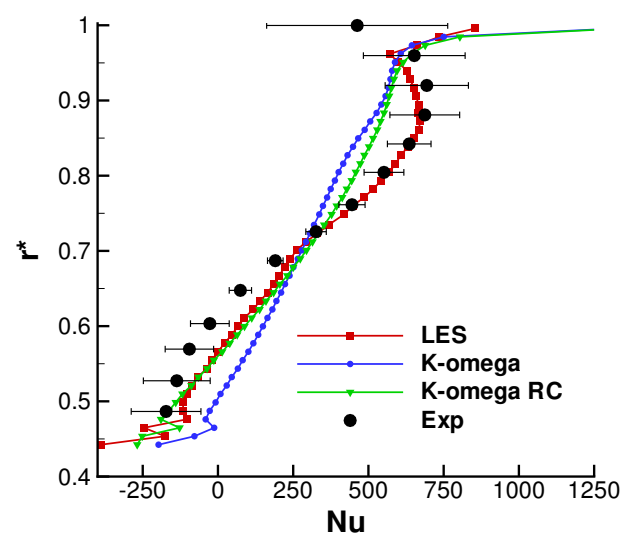

Figure 25. Sensitisation for rotation and curvature effects. Variation of time-average local Nusselt numbers $\mathrm{Nu}$ on the heated wall with nondimensional radius $r^{*}=r / b$.

calculated as

$$
P=\tau_{i j}^{R} \frac{\partial U_{i}}{\partial x_{j}}
$$

where $\tau_{i, j}^{R}$ is the Reynolds stress tensor of the resolved scales, and $U_{i}$ is the mean flow component. It can be noted that the $k-\omega S S T R C$ reduces the value of $P-D$ in agreement with the LES solution. Fig. 25 compares the Nusselt number predicted by the different models. At lower radii, the $k-\omega$ SST RC model gives more accurate results than the $k-\omega S S T$ and the Nusselt number becomes almost coincident with that predicted by the LES. However, at outer radii, the correction provides only a marginal improvement, since the buoyancy phenomenon remains unpredicted.

\section{CONCLUSIONS}

We have presented results from a Large-Eddy Simulation conducted for a rotating cavity with a radial inflow introduced from the shroud and heated on one wall. The case study is highly relevant to the design of secondary air systems in gas turbine engines, as it models a typical configuration of a compressor cavity system. Heat transfer predictions have been compared with experimental data available from the literature, and with those obtained using two URANS eddy-viscosity models widely adopted in industry, namely the Spalart-Allmaras and the $k-\omega S S T$.

The work represents one of the first attempts to study this class of flows by LES, and the results obtained are considered 
to be in good agreement with the data. The LES solution has shown a very good agreement in the outer part of the cavity, capturing buoyancy effects arising due to the interaction between the cold radial inflow and the hot wall. The agreement at inner radii remains good, despite the fact that the resolution employed is not optimal for this region.

Overall, the two URANS models might be said to have given a reasonable agreement with the measured distribution of the Nusselt number, although the results are considerably worse than the LES, especially in the source region. This behaviour is attributed to an excess of turbulent viscosity produced within the rotating core, rendering the turbulent diffusion dominant over the radial convection. This has been shown to affect the structure of the source region, ultimately preventing any buoyancy effect from occurring. It is important to point out that the relevant scaling parameters $\left(R e_{\theta}\right.$ and $\left.C_{w}\right)$ employed in the current study are still far from typical engine conditions. The nondimensional mass flow rate, in particular, could be an order of magnitude larger in an engine, rendering the resolution requirements for an LES much more stringent. With the purpose of making similar calculations usable for design, future work should aim at testing hybrid URANS/LES modeling, along with more mature high-order schemes for industrial codes.

\section{ACKNOWLEDGMENT}

Funding for the work and permission to publish from Rolls-Royce plc are gratefully acknowledged.

\section{REFERENCES}

[1] Kilfoil A.S.R. and Chew J.W., 2009. "Modelling of Buoyancy-Affected Flow in Co-Rotating Disc Cavities", ASME Paper No. GT2009-59214, pp. 1113-1122.

[2] Farthing P.R., Long C.A., Owen J.M. and Pincombe J.R., 1992. "Rotating Cavity with Axial Throughflow of Cooling Air: Flow Structure", Journal of Turbomachinery, Vol. 114(1), pp. 237-246.

[3] Bohn D., Ren J. and Tuemmers C., 2006. "Investigation of the Unstable Flow Structure in a Rotating Cavity", ASME Paper No. GT2006-90494, pp. 1433-1442.

[4] Günther A., Uffrecht W. and Odenbach S., 2012. "Local Measurements of Disk Heat Transfer in Heated Rotating Cavities for Several Flow Regimes", Journal of Turbomachinery, Vol. 134(5).

[5] Tian S., Tao Z., Ding S. and Xu G., 2004. "Investigation of Flow and Heat Transfer in a Rotating Cavity with Axial Throughflow of Cooling Air", ASME Paper No. GT200453525, pp. 373-380.

[6] Sun Z., Lindblad K., Chew J.W. and Young C., 2007. "LES and RANS Investigations into Buoyancy-Affected Convection in a Rotating Cavity with a Central Axial Throughflow", Journal of Engineering for Gas Turbine and Power, Vol. 129(2), pp. 318-325.

[7] Puttock-Brown M.R. and Rose M.G., 2018. "Formation and Evolution of Rayleigh-Bénard Streaks in Rotating Cavities", ASME Paper No. GT2018-75497.

[8] Owen M.J., Abrahamsson H. and Lindblad K., 2007. "Buoyancy-Induced Flow in Open Rotating Cavities", Journal of Engineering for Gas Turbine and Power, Vol. 129(4), pp. 893-900.

[9] Batchelor G. K., 1967. “ An Introduction to Fluid Dynamics”, Cambridge University Press.

[10] Firouzian M., Owen J.M., Pincombe J.R. and Rogers R.H., 1986. "Flow and Heat Transfer in a Rotating Cylindrical Cavity with a Radial Inflow of Fluid, Part 2: Velocity, Pressure and Heat Transfer Measurements", International Journal of Heat and Fluid Flow, Vol. 7(1), pp. 21-27.

[11] Farthing P.R., 1988. "The Effect of Geometry of Flow and Heat transfer in a Rotating Cavity”, Ph.D. thesis, University of Sussex, UK.

[12] Morse A. P., 1988. "Numerical Prediction of Turbulent Flow in Rotating Cavities", Journal of Turbomachinery, Vol. 110(2), pp. 202-211.

[13] Atkins N.R., 2013. "Investigation of a Radial-Inflow Bleed as a Potential for Compressor Clearance Control", ASME Paper No. GT2013-95768.

[14] Sun Z., Amirante D., Chew J.W. and Hills N.J., 2016. "Coupled Aerothermal Modeling of a Rotating Cavity with Radial Inflow", Journal of Engineering for Gas Turbine and Power, Vol. 138(3), pp. 032505.

[15] Amirante D., Sun Z., Chew J.W., Hills N.J. and Atkins N.R., 2016. "Modeling of Compressor Drum Cavities with Radial Inflow", ASME Paper No. GT2016-56505.

[16] Kumar V., Chew J.W., Hills N.J., 2013. "Rotating Flow and Heat Transfer in Cylindrical Cavities with Radial Inflow", Journal of Engineering for Gas Turbines and Power, Vol. 135(3), pp. 032502.

[17] Farthing P.R., Long C.A. and Rogers R.H., 1991. "Measurements and Prediction of Heat Transfer from Compressor Dises With a Radial Inflow of Cooling Air", ASME Paper No. 91-GT-053.

[18] Menter F. R., 1994. "Two-equation eddy-viscosity turbulence models for engineering applications", AIAA Journal, Vol. 32(8), pp.1598-1605.

[19] Spalart P. and Allmaras S., 1992. "A one-equation turbulence model for aerodynamic flows", 30th Aerospace Sciences Meeting and Exhibit, Reno, NV, U.S.A..

[20] Owen J.M. and Rogers R.H., 1995. "Flow and Heat Transfer in Rotating Disc Systems-Rotating Cavities", Vol. 2, Research Studies Press Ltd., London.

[21] Childs P.R.N., 2011. "Rotating Flow”, Elsevier. 
[22] Onori M., Amirante D., Hills N. J. and Chew. J. W., 2017. "LES validation for a rotating cylindrical cavity with radial inflow", ASME Paper No. GT2016-56393.

[23] Owen J. M., Pincombe J. R. and Rogers R. H., 1985. "Source-sink flow inside a rotating cylindrical cavity", Journal of Fluid Mechanics, Vol. 155, pp. 233-265.

[24] Moinier P., 1999. "Algorithm developments for an unstructured viscous flow solver", Ph.D. thesis, Oxford University, Oxford, UK.

[25] Amirante D. and Hills N. J., 2015. "Large-Eddy Simulations of Wall Bounded Turbulent Flows Using Unstructured Linear Reconstruction Techniques", Journal of Turbomachinery, 137(5), pp. 051006.

[26] Hirsch C., 1990. "Numerical Computation of Internal and External Flows", John Wiley and Sons.

[27] Nicoud F. and Ducros F., 1999. "Subgrid-Scale Stress Modelling Based on the Square of the Velocity Gradient Tensor", Flow, Turbulence and Combustion, Vol. 62(3), pp. 183-200.

[28] Dacles-Mariani J., Zilliac G. G.,Chow J. S. and Bradshow P., 1995. "Numerical/Experimental Study of a Wingtip Vortex in the Near Field", AIAA Journal, Vol. 33(9), pp. 1561-1568.

[29] Poinsot T. J. and Lele S. K., 1992. "Boundary Conditions for Direct Simulations of Compressible Viscous Flows", Journal of Computational Physics, Vol. 101(1), pp. 104129.

[30] Davidson L., 2009. "Large Eddy Simulations: How to Evaluate Resolution", International Journal of Heat and Fluid Flow, Vol. 30(5), pp. 1016-1025.

[31] Wilcox D. C. and others, 1998. "Turbulence modeling for CFD”, DCW Industries La Canada.

[32] Hellsten A., 1998. "Some Improvements in Menter's komega SST Turbulence Model”, AIAA paper. 